\title{
Avaliação dos Principais Achados Ultrassonográficos de Esteatose Hepática em Pacientes do Grupo Pós-operatório de Cirurgia Bariátrica do HC Unicamp
}

\section{Ademar Yamanaka, Andrea Saori Hosoda Henriques*, Elinton Adami Chaim, Fábio Henrique Mendonça Chaim.}

\section{Resumo}

A esteatose hepática $(\mathrm{EH})$ é definida como distúrbio do metabolismo caracterizada pela infiltração gordurosa do fígado(1,2). Estudos apontam uma forte associação entre obesidade mórbida e a incidência de EH e doenças crônicas(3). O trabalho tem como objetivo principal realizar um estudo retrospectivo de imagens ultrassonográficas (US) e de 50 prontuários de pacientes do grupo pós-operatório de cirurgia bariátrica do Hospital de Clínicas da UNICAMP para verificar se a classificação de EH por características de imagem presentes na literatura condizem com os dados encontrados em pacientes que foram submetidos à cirurgia bariátrica.

\section{Palavras-chave:}

Ultrassonografia, esteatose hepática, cirurgia bariátrica.

\section{Introdução}

A esteatose hepática $(E H)$ é definida como distúrbio do metabolismo caracterizada pela infiltração gordurosa do fígado(1,2). Estudos apontam uma forte associação entre obesidade mórbida e a incidência de $E H$ e doenças crônicas como Diabetes Mellitus tipo 2(DM2), hipertensão arterial sistêmica(3). A EH pode ocorrer de forma benigna ou evoluir para formas mais severas, como exemplo a esteato-hepatite e cirrose hepática, o que leva pacientes com obesidade a terem um maior risco de evolução a óbito (4). A cirurgia bariátrica já demonstrou diversos benefícios como a diminuição de doenças crônicas e neoplásicas. (5). Embora hajam diversas classificações acerca dos graus de $\operatorname{EH}(6,7)$, ainda é preciso esclarecer melhor o comportamento destes parâmetros ultrassonográficos em pacientes com obesidades graus I, II e III.

O trabalho tem como objetivo principal realizar um estudo retrospectivo de imagens ultrassonográficas (US) e de 50 prontuários de pacientes participantes do grupo pós-operatório de cirurgia bariátrica do Hospital de Clínicas da UNICAMP para verificar se a classificação de EH por características de imagem presentes na literatura condizem com os dados encontrados em pacientes que foram submetidos à cirurgia bariátrica, além de realizar uma análise do perfil hepático dos pacientes em diferentes tempos de pós-operatório.

\section{Resultados e Discussão}

De acordo com os dados levantados a partir de referências bibliográficas lidas até o momento, a presença dos parâmetros de Contraste Hepatorrenal $(\mathrm{CHR})$ e Contraste Hepatoesplênico(CHE), quando presentes, são considerados preditores para diagnosticar $\mathrm{EH}$. Isso ocorre devido ao fato de que a velocidade do parênquima hepático, córtex renal e baço são bem próximas em pacientes normais (6). Porém, na prática diária de US, são notados pacientes com EH que apresentam aumento da ecogenicidade tanto no fígado quanto no parênquima renal e esplênico, resultando em ausência de contraste. Isso traz a hipótese de que o acúmulo de gordura ocorre não somente no fígado, como também no baço e no parênquima renal. Sendo essa hipótese verdadeira, os parâmetros de $\mathrm{CHR}$ e $\mathrm{CHE}$ não teriam boa sensibilidade para diagnosticar a esteatose hepática e por isso torna-se importante o estudo de outros parâmetros (Classificação por Atenuação Sonora, Veias Hepáticas e Borda Hepática) em casos de ausência de CHR e CHE.

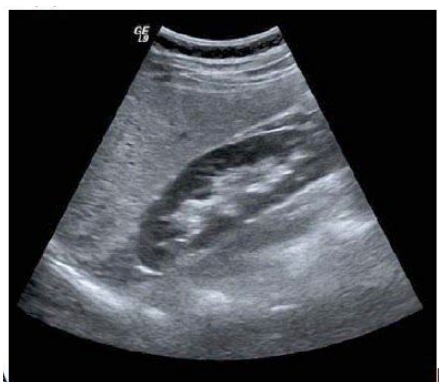

Figura 1. Imagem Ultrassonográfica demonstrando ecogenicidade distinta entre o fígado esteatótico e parênquima renal direito $(\mathrm{CHR})(7)$.

\section{Conclusões}

Aplicando-se o teste Qui Quadrado com os dados obtidos de CHR e CHE e comparando-os com os outros parâmetros (Classificação por Atenuação Sonora, Veias Hepáticas e Borda Hepática), será possível avaliar se os parâmetros de $\mathrm{CHR}$ e $\mathrm{CHE}$, mesmos quando ausentes, podem indicar esteatose hepática, evidenciando assim sua sensibilidade reduzida para diagnóstico de $\mathrm{EH}$.

1. Machado MM, Rosa AC, Cerri GG. Doenças hepáticas difusas, hipertensão portal e transplante de fígado. In: Cerri GG, editor. Ultra-sonografia abdominal. Rio de Janeiro, RJ: Revinter; 2002. p. 55-124.

2. Gayotto LC, Comitê SBP/SBH. Visão histórica e consenso nacional sobre a classificação das hepatites crônicas. GED. 2002;19:137-40.

3. Jorge, Liziara Cardoso; Araújo, Mariana Silva Melendez., Influência da Cirurgia Bariátrica do DM2 e outras comorbidades: revisão de literatura / Influence of Bariatric Surgery in DM2 and other comorbidities: literature review Comun. ciênc. saúde, ago. 2012; 23(1):67-72.

4. LEVENE A.P. \& GOLDIN R.D. The epidemiology, pathogenesis and histopathology of fatty liver disease. Histopathology, 2012. v. 61, n. 1, p. $141-152$.

5. Weiner RA. Surgical treatment of non-alcoholic steatohepatitis and non-alcoholic fatty liver disease. Dig Dis. 2010;28(1):274-9.

6. Yamanaka, et al. Atlas de ultrassonografia abdominal: Achados normais e patológicos. 1 ed. Campinas: Unième, 1996; pág. 85.

7. Monjardim RF, Costa DMC, Romano RFT, Salvadori PS, Santos JVC, Von Atzingen AC, Shigueoka DC, D'Ippolito G. Diagnóstico da esteatose hepática pela tomografia computadorizada de abdome com meio de contraste intravenoso. Radiol Bras. 2013 Mai/Jun;46(3): 134-138. 